\title{
The Trojan Citation and the "Accidental" Plagiarist
}

\author{
David Shaw
}

Received: 3 September 2015 / Accepted: 14 December 2015 / Published online: 16 January 2016

(C) Journal of Bioethical Inquiry Pty Ltd. 2016

Keywords Plagiarism · Authorship · Research integrity Scientific misconduct $\cdot$ Publishing ethics

Imagine a researcher's horror when he comes across a paper that was published a year after one of his own and presents almost exactly the same theories and ideas. This must be plagiarism! But when he checks the reference list, he realizes that his original work is actually citedonce and only in relation to a minor point. Many other ideas are copied without credit. Is this plagiarism or just bad citation? Unfortunately, it is very unlikely to be the latter. This scenario is a result of an unpleasant phenomenon in academia: the Trojan citation, which makes it appear that all is well, but actually hides several major instances of plagiarism. Although it is not yet clear how widespread this phenomenon is, both journal editors and researchers should be aware of the dangers of this weapon and the stealthy (or perhaps accidental) plagiarist who wields it.

Someone who uses a Trojan citation makes reference to a source one time to in order to evade detection (by editors and readers) of bad intentions and provide cover for a deeper, more pervasive plagiarism. In a sense, this makes the original author something of a "ghost author" of the newer paper, as his contribution to it goes unacknowledged. The Trojan citation is not in itself plagiarism but rather an attempt to dismiss charges of

D. Shaw $(\bowtie)$

Institute for Biomedical Ethics, University of Basel,

Bernoullistrasse 28, 4056 Basel, Switzerland

e-mail: david.shaw@unibas.ch plagiarism; on a basic reading, if the original work is cited, then it isn't plagiarism. This makes the use of a Trojan citation a much subtler tactic than "mosaic plagiarism," where plagiarism of ideas is used alongside direct replication of text (Das and Panjabi 2011). Mosaic plagiarism is quite common (Das and Panjabi 2011), but it is easy to detect because the copying of original text appears alongside the new author's own phrases. In contrast, the more sophisticated Trojan citation involves neither direct copying of text nor total failure to cite the source. Like the Trojan Horse, which appears benign but hides great danger, the Trojan citation provides a veneer of respectability to the theft of ideas by acknowledging part of the intellectual debt in order to hide plagiarism elsewhere in the work.

But the strategy of the plagiarist who deliberately deploys the Trojan citation also has its disadvantages. Had no reference been provided, any charge of plagiarism could then be answered with "sheer coincidence, I never read that other paper" (suspicious though such a response might seem). Providing the reference allows the discerning reader (or indeed the author of the original piece) to draw direct comparisons. If all the major points in a paper are also made in an earlier one, but the original paper is only cited in support of one of the points, this is clearly plagiarism. Of course, the Trojan plagiarist, if challenged, might well say that it was simply careless referencing, but that excuse would not be accepted from a first-year undergraduate, much less a seasoned academic. The plagiarist might also reply that the one citation was intended to imply that all the arguments in the newer work were based upon the old, which in turn raises several rather awkward questions: if 
you knew that, then (a) why not cite properly and (b) why submit the paper at all if it was so unoriginal? It is very implausible that a suspected case of Trojan citation could be due to accidental referencing rather than deliberate deceit; even if it is, poor citation also indicates a lack of research integrity.

Another excuse employed by the author who uses the Trojan citation (which recently occurred in an actual case, where a colleague identified similarities and challenged the individual) is that he actually had the main ideas himself and belatedly realized that a very similar paper had already been published just before submitting his own work. This feeble pretext also raises some difficult questions. If true, this implies that the Trojan author's use of literature must have been sparing at best; how could such similar work not be detected with a basic search of previous research? Furthermore, any researcher with integrity would either abandon the paper on finding out that someone else already published it or rigorously cite every claim or argument in the paper that had already been made elsewhere. Yet doing the first of these would certainly rob the Trojan author of a publication, and the second would very likely decrease his chances, because it would amount to admitting that the content of the new paper was not original. In this sense, of course, the Trojan-citing plagiarist commits an offence not only against the original author but also against the journal in question - and against all academic endeavour.

Of course, this is all assuming that the plagiarist didn't simply paraphrase an entire paper and then add in a Trojan citation in order to be able to make the excuse about "throwing in a late reference." While doing so might seem disingenuous, a journal is much more likely to take seriously a claim that ideas have been plagiarized when there is no citation. If a newer paper uses the same ideas as an old one, and does not cite it, plagiarism is quite obvious; in contrast, if a Trojan citation is provided, it clouds the issue and could make it look like there is no plagiarism.

I have experienced this personally. In 2013, a colleague and I published a commentary article in a leading medical journal that made several points about patient interaction. Around a year later, a very similar article appeared in a rival medical journal that made the same main arguments. At first, I was outraged that someone had copied our work, but I then realized that our paper was actually listed in the references. The body of the text, however, cited our paper only in relation to one minor point, while our other arguments-clearly reiterated - were not cited at all. When I pointed these similarities out to the journal's editors, with detailed quotations from our original paper, the response was: "We take allegations of plagiarism very seriously. We understand plagiarism as only the verbatim copying of someone else's work without making it clear and giving credit." The latter sentence somewhat contradicts the first; this is an exceedingly narrow definition of plagiarism. The international definitions to which the journal in question is supposed to adhere define it much more broadly. The Committee on Publication Ethics (COPE) defines plagiarism as "[w]hen somebody presents the work of others (data, words or theories) as if they were his/her own and without proper acknowledgment" (COPE n.d., "1), while the World Association of Medical Editors (WAME) explains it as "the use of others' published and unpublished ideas or words (or other intellectual property) without attribution or permission, and presenting them as new and original rather than derived from an existing source" (WAME 2015, 『1 under "Plagiarism"). Despite claiming to adhere to COPE's rules, the journal's editors concluded that "[f]rom the examples you have given it seems there is no case of plagiarism to answer here as we understand it because the text is substantially different even if the ideas are similar. And your article is cited."

This example illustrates the conflict of interest that journals and their editors face in responding to allegations of plagiarism involving Trojan citations. In unambiguous cases where text is copied without citation, plagiarism is obvious and they must respond. In cases where ideas are used without any attribution, there may also be a case to answer, even if the author dismisses it as coincidence. But in cases where one component is cited but others are not, the Trojan citation provides sufficient cover for both the author and the journal to dismiss the concerns - even if the journal in question claims to take plagiarism seriously. If the journal admits that there is a problem, it is essentially admitting that its peer review or editorial review system failed to detect that an article was a rehash of other recent work on exactly the same topic.

Part of the problem may be that ideas are fuzzier than words; direct copying of text without attribution is obvious and easy to spot, but it is rather more difficult (but not impossible) to identify copied concepts. The editors in this case erroneously claimed that "[o]f course authors should give credit where it is due in any case, but there is no copyright on ideas. It is inevitable that ideas 
get reiterated, repeated and reformulated, and who is to say in general who had the thought first or what inspired an idea?" One hopes that editors in the humanities and social sciences, where concepts are perhaps treated with greater respect than in data-driven disciplines like medicine, would have known that the obvious answer is "the publication date of the earlier paper establishes who has priority." The definitions above make it clear that ideas are also supposed to be protected from plagiarism, even if a Trojan citation is used to deflect accusations of misconduct. Although I only have my own experience to draw on, the fact that two of my seventy published papers have already been victims of Trojan citations may be indicative of a wider trend.

The Trojan citation represents a new challenge for journals. Whether it indicates poor referencing (which is still unacceptable) or a genuine attempt to claim credit for someone else's ideas will depend on the case in question, but editors must remain vigilant for Trojan citations - both in assessing an article's originality when it is submitted and in responding to cases of suspected plagiarism. In many cases, detection of Trojan citations will require intimate knowledge of the literature, so only specialist reviewers may be expert enough to detect them. (Indeed, in the two cases I noticed, I only did so because it was my work that was involved.) If editors dismiss concerns simply because the original author's work is cited once, they are complicit in research misconduct, whether by accident or design. The Trojan citation is a relatively new weapon in the armoury of the unethical author, but journal editors and reviewers will hopefully soon learn how to identify this tactic and take any necessary action. Researchers, too, should be alert for Trojan citations and alert journals to any suspicion of "accidental" plagiarism.

\section{References}

Das, N., and M. Panjabi. 2011. Plagiarism: Why is it such a big issue for medical writers? Perspectives in Clinical Research 2(2): 67-71.

Committee on Publication Ethics (COPE). No Date. Plagiarism. http://publicationethics.org/category/keywords/plagiarism. Accessed December 7, 2015.

World Association of Medical Editors (WAME). 2015. Recommendations on publication ethics policies for medical journals. http://www.wame.org/about/recommendations-onpublication-ethics-policie. Accessed December 7, 2015. 